\title{
Anterolateral Necrotic Lesion Serves as an Early Warning Sign of Developing Collapse in Osteonecrosis of the Femoral Head
}

\section{Qiu-Shi Wei}

the Third Affiliated Hospital of Guangzhou Unversity of Chinese Medicine

\section{Min-Cong He}

Guangzhou Research Institute for Orthopedics \& Traumatology of Chinese Medicine

\section{Xiao-Ming He}

Guangzhou Research Institute for Orthopedics \& Traumatology of Chinese Medicine

Tian-Ye Lin

Guangdong Research Institute for Orthopedics \& Traumatology of Chinese Medicine

\section{Peng Yang}

Guangdong Research Institute for Orthopedics \& Traumatology of Chinese Medicine

\section{Zhen-Qiu Chen}

First affiliated Hospital of Guangzhou University of Chinese Medicine

\section{Qing-Wen Zhang}

The Third Affiliated Hospital of Guangzhou University of Chinese Medicine

Wei He ( $\nabla$ hewei13802516062@163.com)

The Third Affiliated Hospital of Guangzhou University of Chinese Medicine

\section{Research article}

Keywords: Osteonecrosis of the femoral head, Collapse, Anterolateral, Necrotic lesion

Posted Date: July 8th, 2021

DOl: https://doi.org/10.21203/rs.3.rs-676727/v1

License: (1) (1) This work is licensed under a Creative Commons Attribution 4.0 International License. Read Full License 


\section{Abstract}

Objective: Load bearing capacity of the bone structure of anterolateral weight-bearing area plays an important role in the progressive collapse in osteonecrosis of the femoral head (ONFH). The purpose of this study is to assess the efficacy of combined evaluation of anteroposterior (AP) and frog-leg lateral (FLL) view to diagnose collapse.

Methods: Between December 2016 to August 2018, a total of 478 hips from 372 patients with ONFH (268 men, 104 women; mean age $37.9 \pm 11.4$ years) were retrospectively evaluated. All patients received standard AP and FLL views of hip joints. Japanese Investigation Committee (JIC) classification system was used to classified necrotic lesion in AP view. Anterior necrotic lesion was evaluated by FLL view. All patients with precollapse ONFH underwent non-operative hip preserving therapy. The collapse rate was calculated and compared with Kaplan-Meier survival analysis with radiological collapse as endpoints.

Results: Forty-four (44/478, 9.2\%) hips were classified as type A, $65(65 / 478,13.6 \%)$ as type B, 232 (232/478, 48.5\%) as type C1, and $137(137 / 478,28.7 \%)$ as type C2. Three hundred cases (300/478, $62.5 \%)$ were collapsed at the initial time point. Two hundred and twenty six $(226 / 300,75.3 \%)$ hips and 298 (298/300, 99.3\%) hips collapse were identified with AP view and FLL view, respectively. An average follow-up of $37.0 \pm 32.0$ months was conducted to evaluate the occurence of collapse in 178 precollapse hips. During follow-up period, collapse occurred in 89 hips (50.0\%). Seventy-seven (77/89, 86.5\%) hips was determined with AP view alone and 85 (85/89, 95.5\%) hips were determined with combination of AP and FLL views. The collapse rate at five years were reported as $0 \%$ and $0 \%, 16.2 \%$ and $24.3 \%, 58.3 \%$ and $68.1 \%$, and $100 \%$ and $100 \%$ according to combination of AP and FLL views or AP view alone for types A, $\mathrm{B}, \mathrm{C} 1$, and $\mathrm{C} 2$, respectively.

Conclusion: The collapse can be diagnosed more accurately by combination of AP and FLL views. Besides, JIC type A and type B ONFH can be treated with conservative hip preservation, but precollapse type $\mathrm{C} 2 \mathrm{ONFH}$ should be treated with joint-preserving surgery. Type $\mathrm{C} 1$ needs further study to determine which subtype has potential risk of collapse.

\section{Introduction}

Osteonecrosis of the femoral head (ONFH) is a devastating disease in young patients and generally progresses to collapse of the femoral head and secondary hip osteoarthritis ${ }^{1}$. Hip pain and loss of function can affect the quality of life resulting in the need of total hip arthroplasty ${ }^{2}$. Hip preservation is the preferred therapy for young patients. Femoral head collapse is the most significant clinical turning point of ONFH. Once collapse occurs, the effect of hip preservation is greatly reduced ${ }^{3}$. Therefore, accurate prediction and evaluation of collapse risk in the precollapse stage can help to identify those patients who might benefit from intervention to prevent collapse ${ }^{4}$. 
Femoral head collapse is closely linked to the size and location of the necrotic lesion ${ }^{5-9}$. Especially, a large-sized necrotic lesion and lateral lesion of the femoral head are two major causes of femoral head collapse ${ }^{5}$. Although some reports have attempted to evaluate the collapse risk of ONFH focusing on the area or volume of necrotic lesion, these studies have limitations in terms of small sample sizes and lacking of consistency in clinical application ${ }^{7 ; 8}$. Recently, Cheng et al indicates that the location of the necrotic lesion is considered as the highest risk factors. Necrotic lesion located on the lateral and anterior femoral head had high collapse risk, as shown in using magnetic resonance imaging (MR), computed tomography (CT), or radiographs ${ }^{10-12}$. The anterior and lateral bone structure of the femoral head is a critical weight-bearing area and the load-bearing ability reduced as a result of impairment of necrotic bone ${ }^{13-17}$. Therefore, an ideal method for predicting the progression of ONFH should focus on evaluating the bone structure in the weight-bearing area. An appropriate treatment to the weaken anterolateral weight-bearing area of the femoral head was essential for those in high risk of collapse ${ }^{18}$.

The ability to easily predict femoral head collapse based on plain radiological types would be valuable and preferable for clinical use, especially in developing countries ${ }^{19}$. Although the Japanese Investigation Committee (JIC) classification which is defined according to AP view is proven to be a reliable system to predict the collapse risk ${ }^{12}$, the limitation is that $\mathrm{JIC}$ may overlook collapse exiting on the anterior femoral head. Some cases with necrotic lesions occupying more than the medial two-thirds of the weight-bearing area behave as an intact articular surface in AP view but a significant collapse was found in FLL view ${ }^{20}$. This situation reminds us a combined evaluation of AP and FLL views might be more accurate to assess the status of a necrotic femoral head.

The purpose of this study was to estimate and compare the collapse rate by using the AP view alone and a combination of AP and FLL views and develop personalized therapeutic strategies for patients with precollapse ONFH.

\section{Materials And Methods}

This retrospective cohort study have included 478 hips from 372 patients (268 men, 104 women) diagnosed with ONFH between December 2016 to August 2018. Clinical data and radiographic information were collected. This study was approved by the ethics committee of the Third Affiliated Hospital of Guangzhou University of Chinese Medicine (NO. PJ-KY-20210401-001).

All patients were diagnosed with ONFH and classified into Association Research Circulation Osseous (ARCO) staging system ${ }^{21}$ and the JIC classification system ${ }^{12}$. The inclusion criteria were as follows: (1) above the age of $14,(2)$ with AP and FLL views of both hip joints, (3) agreed to perform conservative treatment, and (4) follow-up of $>24$ months. The exclusion criteria were as follows: (1) accompanying with serious diseases, such as dysplasia of the hip joint, rheumatoid arthritis, ankylosing spondylitis, joint tuberculosis, or pyogenic arthritis, (2) absence of clinical or radiographic data, and (3) previously underwent hip-preserving surgery. Demographic, radiological, and surgical intervention data from 
Orthopaedics Database System v1.0 was collected which had obtained the copyright in computer software (2017SR274625) registered by the State Copyright Administration. The demographic data have included age, sex, laterality, etiology (steroid use, alcohol intake, idiopathic disease, or traumatic), duration of symptom (asymptomatic, pain duration of hip $\leq 6$ months, and $>6$ months), and surgical procedure (if relevant). Radiological data have included the stage of ONFH at initial diagnosis and radiological change(s) during the follow-up.

\section{Radiological evaluation}

AP and FLL views of both hip joints were performed at the time of initial diagnosis. All plain radiographs were taken using standardized techniques. For the AP view, the patient was positioned supine on the x-ray table and bilateral legs abducted in neutral position so that the distance between the two feet is equal to shoulder width. For the FLL view, the patient was positioned supine on the $x$-ray table and the bilateral hips was flexed at a degree of $30^{\circ}$. The thigh was abducted and externally rotated while ensuring that the feet contacted close to each other at the level of ipsilateral knee. The x-ray beam was directed anterior to posterior and centered on the femoral head while the plane of the pelvis was parallel to the plane of the table (Fig. 1) ${ }^{22}$.

The stage of ONFH was defined according to the ARCO staging system ${ }^{21}$ : stage I is identified as a normal radiograph with an abnormal MRI or bone scan; stage II is identified as cystic and sclerotic changes in the femoral head but without any evidence of subchondral fracture or flattening of the femoral head; stage III is identified as a crescent sign and/or flattening of the articular surface, this stage is further divided into IIIA (collapse $\leq 2 \mathrm{~mm}$ ) and IIIB (collapse $>2 \mathrm{~mm}$ ); and, stage IV is identified as the appearance of degenerative changes with accompanying joint space narrowing, and/or joint destruction. The degree of the collapse was also measured using concentric circles on both AP and FLL views using the Image $\mathrm{J}$ program ${ }^{11}$. This study have included patients in ARCO stage II and III and signs of necrotic zone, sclerosis change and/or collapse could be seen on X-ray clearly. Consequently, the JIC type was determined basing on the AP and FLL views. The JIC classification comprises four types according to necrotic location: $A, B, C 1$, and $C 2{ }^{12}$. Type $A$ represents necrotic lesion is located in the medial one-third of the weight-bearing surface relative to acetabular eyebrow arch; type $B$ involves medial two-thirds of the weight-bearing surface; type $\mathrm{C} 1$ lesions are characterized by a necrotic zone that spans more than the medial two-thirds of the weight-bearing surface at the acetabular edge; type $\mathrm{C} 2$ involves the necrotic zone that spans more than the lateral one-third of the weight-bearing surface and exceeds the acetabular edge. To establish reliability, all image data measurements were made by two experienced orthopedic surgeons in a blinded manner.

Nonoperative management was recommended to patients with ARCO stage II ONFH. These conservative treatments consisted of pain relief, restricted weight bearing, and physical therapy. Restricted weightbearing was maintained with the use of two crutches for the first 3 months after initial diagnosis. Partial weight-bearing using one crutch was recommended in the next following 3 months. Then, full weightbearing was allowed when the patient was free of hip pain. All patients underwent clinical and 
radiographic examination at each 3 or 4 month during follow up for the first 2 years and then receive annually examination thereafter. Demographic features, ARCO stage, JIC type, and collapse or not on FLL view were recorded at initial diagnosis. Above data information in patients with ARCO stage III ONFH also were collected before treatment.

\section{Statistical analysis}

Statistical analyses were performed by using the SPSS version 13.0 software (SPSS Inc., USA). Chisquared test was used to compare the laterality, etiology, JIC type, and duration of symptom. Further, Kaplan-Meier survival analysis was performed to calculate the collapse rates of the femoral head and collapse was set as the endpoint. A comparison of the Kaplan-Meier curves for the relevant factors (JIC type, etiology, and onset of symptom) was performed with a log-rank test in the precollapse cohort. A cox regression model was used to evaluate the independent prognostic factors associated with collapse, including JIC type (AP view only or combination with FLL view), etiology, and duration of pain. All statistical analyses were two-sided and evaluated with a p-value of less than 0.05 indicating statistical significance.

\section{Results}

\section{Demographics}

Between December 2016 to August 2018, 478 hips from 372 patients with ONFH were reviewed. At initial diagnosis, 178 hip in 135 patients including 93 male and 42 female with a mean age of $40.4 \pm 11.5$ years (range, 17-64) were defined as ARCO stage II. A number of 300 hips in 237 patients including 175 male and 62 female with a mean age of $36.21 \pm 10.8$ years (rang, 14-62) were defined as ARCO stage III with stage IIIA 197 hips and IIIB103 hips, respectively. According to the JIC types, 44 hips were classified as type A, 65 hips type B, 232 hips type C1, and 137 hips type C2. The demographic, clinical, and radiographic characteristics are shown in Table 1. 
Table 1

Demographic data

\begin{tabular}{|c|c|}
\hline Parameters & $\mathbf{N}$ \\
\hline Number of patients/hips & $372 / 478$ \\
\hline Mean age at initial diagnosis, years(range) & $37.9 \pm 11.4(14-63)$ \\
\hline Gender (Males/Females) & $268 / 104$ \\
\hline Side (Left/Right) & $242 / 236$ \\
\hline Bilateral:unilateral & $106: 266$ \\
\hline \multicolumn{2}{|l|}{ Etiology† } \\
\hline Steroid & 186 \\
\hline Alcohol & 123 \\
\hline Idiopathic & 41 \\
\hline Trauma & 22 \\
\hline \multicolumn{2}{|l|}{ ARCO stage (Baseline) } \\
\hline ૧ & 178 \\
\hline$\nabla \mathrm{A}$ & 197 \\
\hline$\nabla \mathrm{B}$ & 103 \\
\hline \multicolumn{2}{|l|}{ Location (Baseline) } \\
\hline A & 44 \\
\hline B & 65 \\
\hline $\mathrm{C} 1$ & 232 \\
\hline $\mathrm{C} 2$ & 137 \\
\hline \multicolumn{2}{|l|}{ Onset of symptom } \\
\hline Asymptomatic & 79 \\
\hline$<=6$ months & 255 \\
\hline$>6$ months & 144 \\
\hline
\end{tabular}

\section{Collapse rate at initial diagnosis}

As shown in Table 2, there were no significant differences in collapse rate between left and right sides $\left(\mathrm{X}^{2}\right.$ $=0.000, p=0.982)$. There was also no significant difference between patients with and without steroid 
use $\left(X^{2}=0.142, p=0.706\right)$. There were significant differences in terms of the collapse rate among four JIC types $\left(X^{2}=291.154, p<0.001\right)$. The collapse rates in types $A, B, C 1$, and $C 2$ were $2.5 \%, 43.1 \%, 69.0 \%$, and $77.4 \%$, respectively. Moreover, there was also a significant difference in collapse rate at initial diagnosis between asymptomatic and symptomatic hips $\left(X^{2}=92.077, p<0.001\right)$. The collapse rates of asymptomatic hips were significantly lower $(15.2 \%)$ than those of symptomatic hips $(72.2 \%)$.

Table 2

Collapse rate at initial diagnosis as analyzed by demographic data.

\begin{tabular}{|c|c|c|c|c|c|c|}
\hline \multirow[t]{2}{*}{ Parameter/stage } & \multicolumn{3}{|c|}{ ARCO stage } & \multirow[t]{2}{*}{$\mathrm{n}$} & \multirow[t]{2}{*}{ Collapse hips at initial diagnosis, n (\%) } & \multirow[t]{2}{*}{$p$-value } \\
\hline & II & IIIA & IIIB & & & \\
\hline \multicolumn{6}{|l|}{ Side (Left/Right) * } & \multirow{3}{*}{$0.982^{\#}$} \\
\hline Left & 90 & 101 & 51 & 242 & $152 / 242(62.8 \%)$ & \\
\hline Right & 88 & 96 & 52 & 236 & $148 / 236(62.7 \%)$ & \\
\hline \multicolumn{6}{|l|}{ Etiology* } & \multirow[t]{3}{*}{$0.706^{\#}$} \\
\hline Steroid & 91 & 104 & 56 & 251 & $159 / 251(63.3 \%)$ & \\
\hline Non-Steroid & 87 & 93 & 47 & 227 & $140 / 227(61.7 \%)$ & \\
\hline \multicolumn{6}{|c|}{ Location (Baseline)* } & \multirow[t]{5}{*}{$<0.001^{\#}$} \\
\hline A & 38 & 6 & 0 & 44 & $6 / 244(2.5 \%)$ & \\
\hline B & 37 & 27 & 1 & 65 & $28 / 65(43.1 \%)$ & \\
\hline $\mathrm{C} 1$ & 72 & 118 & 42 & 232 & $160 / 232(69.0 \%)$ & \\
\hline $\mathrm{C} 2$ & 31 & 46 & 60 & 137 & $106 / 137(77.4 \%)$ & \\
\hline \multicolumn{6}{|c|}{ Onset of symptom* } & \multirow[t]{4}{*}{$<0.001^{\#}$} \\
\hline Asymptomatic & 67 & 9 & 3 & 79 & $12 / 79(15.2 \%)$ & \\
\hline$<=6$ months & 74 & 129 & 52 & 255 & $181 / 255(71.0 \%)$ & \\
\hline$>6$ months & 37 & 59 & 48 & 144 & $107 / 144(74.3 \%)$ & \\
\hline \multicolumn{7}{|c|}{ * Values are given as the number of hips. } \\
\hline \multicolumn{6}{|l|}{ \# Chi-Square test. } & \\
\hline
\end{tabular}


Table 3

Radiographical characteristics of patients with collapsed ONFH.

\begin{tabular}{|lll|}
\hline Parameters & \multicolumn{2}{l|}{ ARCO stage } \\
\cline { 2 - 3 } & IIIA & IIIB \\
\hline Number of hips (n) & 197 & 103 \\
\hline AP view & & \\
\hline Femoral head noncollapse (n) & 72 & 2 \\
\hline Femoral head collapse (n) & 125 & 101 \\
\hline Negetive in FLL view (n) & 2 & 0 \\
\hline Collapse rate (\%) & $63.5 \%$ & $98.1 \%$ \\
\hline FLL veiw & & \\
\hline Femoral head noncollapse (n) & 2 & 0 \\
\hline Femoral head collapse (n) & 195 & 103 \\
\hline Negetive in AP viewview (n) & 72 & 2 \\
\hline Collapse rate (\%) & $99.0 \%$ & $100 \%$ \\
\hline Combination of AP and FLL views & $100 \%$ & $100 \%$ \\
\hline Collapse rate (\%) & & \\
\hline ONFH = osteonecrosis of femoral head & \\
\hline
\end{tabular}

\section{Collapse rates observed at initial diagnosis}

In the 300 collapsed-hips, the number of the collapsed femoral head in ARCO stage IIIA found by FLL view was significantly higher than that of by AP view $\left(X^{2}=81.529, p<0.001\right)$. However, there were no significant differences in the collapse rate between two position radiographs $\left(X^{2}=2.020, p=0.155\right)$. In the 197 hips with ARCO stage IIIA, 2 collapsed-hips (1.0\%) were detected in AP view, but not on FLL view. Seventy-two $(72 / 197,36.5 \%)$ collapsed hips were detected in FLL view but not on AP view. Further analysis of this 72 hip indicated that 6 hips were JIC type A, 24 hips type B, 41 hips type C1, and 1 hips type $\mathrm{C} 2$. Necrotic femoral heads were collected after hip replacement, and hard tissue slicing showed a collapse at the anterolateral portion of the femoral head or anterior portion alone (Fig. 2).

\section{Collapse rate assessed by Kaplan-Meier survival analysis}

The risk factors of collapsing in 178 precollapse hips were analysed by log-rank test, and the mean follow-up period was $37.0 \pm 32.0$ months (rang. 1 to 141). The collapse rates at five year were evaluated 
and described as $0 \%$ in type $A, 16.2 \%$ in type $B, 58.3 \%$ in type $C 1$, and $100 \%$ in type $C 2\left(X^{2}=125.574, p<\right.$ 0.001; Fig. 3A); With the definition of JIC types according to FLL view, type A have the collapse rate of $0 \%$; type B 24.3\%; type C1 68.1\%; and type C2 100\% ( $X^{2}=125.030, p<0.001$; Fig. $3 B$ ). In addition, there was no significant difference in steroid and non-steroid groups $\left(X^{2}=1.752, p=0.186\right.$; Fig. $\left.3 C\right)$, and a significant difference was indicated between patients with asymptomatic and symptomatic ONFH $\left(X^{2}=\right.$ 49.613, $\mathrm{p}<0.001$; Fig. 3D).

\section{JIC type may be a collapse-correlated independent risk factor assessed by the Cox regression model}

The cox regression analysis indicated that JIC type was an independent risk factor associated with collapse [p $<0.001$, hazard ratio (HR) $4.551 ; 95 \%$ confidence interval $(\mathrm{Cl}) 3.263$ to 6.348$]$. When FLL view was combined with AP view to determine collapse, a lower HR value was shown [p < 0.001, HR 4.106; 95\% $\mathrm{Cl} 3.039$ to 5.547]. Moreover, the HR value was 4.319 for type $C 1$ compared to type $B(p<0.001)$, and 3.115 for type $\mathrm{C} 2$ compared to $\mathrm{C} 1$ ( $p<0.001$ ) (Fig. 4). In addition, there was no significant association between collapse and other clinical factors such as etiology $(p=0.677)$ and pain duration of hip $(p=$ 0.938).

\section{Surgical treatments}

Surgical treatments was performed for a total of 76 hips (25.3\%) in 300 collapsed-hips, namely, total hip arthroplasty (21 hips), surgical hip dislocation approach combined with anterior iliac bone grafting (31 hips), free vascularized fibular grafting (20 hips), and intra-articular injection of platelet-rich plasma(4 hips). In 178 precollapse hips, 89 hips (50.0\%) were detected collapse during the follow-up period. Surgical treatments was performed for a total of 38 hips (42.7\%), namely, total hip arthroplasty (29 hips), surgical hip dislocation approach combined with anterior iliac bone grafting (4 hips), and free vascularized fibular grafting (5 hips). No surgical intervention was need in all 75 hips with JIC type A and type B during follow-up.

\section{Discussion}

This study reported that the collapse during the progress of ONFH can be determined more accurately by combined evaluation of AP and FLL views. JIC types based on combination of AP and FLL views have a predictable value for collapse according to the survival analysis. The cox regression analysis indicated that JIC types based on the AP view was an independent risk factor for collapse, and when JIC types were defined by combination of AP and FLL views, the collapse risk was higher The results emphasize that the necrotic lesion in anterolateral weight-bearing area of the femoral head should be fully evaluated by not only AP view but also combined with FLL view. This method is simple, low-cost, and easy to perform and repeat reliably. It is appropriate predicting the occurrence of collapse at initial diagnosis and at follow-up.

It is always a controversial topic on optimal treatment for asymptomatic $\mathrm{ONFH}^{23}$. Considering the high collapse rate, some studies proposed early diagnosis and surgical intervention to preserve hip joint in 
ONFH patients even without symptoms ${ }^{24}$. In contrast, several authors recommended surgical treatment just for patients with symptomatic $\mathrm{ONFH}^{25}$. Lacking of reliable data of the collapse rate is the main reason for this controversy.

The extent and location of the necrotic lesion are recognized as risk factors in femoral head collapse ${ }^{5-7}$; $17 ; 20$. Accordingly, there are different kinds of methods basing on the three-dimensional imaging to assess extent and location of the necrotic lesion to predict collapse, such as MRI and CT 7; 26; 27 . Many complicate image data was presented in these methods, and it is hard to reach a consensus in particular which layer todetermine for predicting collapse in MRI or CT scan. In addition, CT scan involves higher doses of radiation and MRI is relatively high cost examination. Therefore, it is generally believed that the ability to easily and accurate predict femoral head collapse based on plain radiological would be valuable and preferable for clinical used, especially in developing countries ${ }^{28}$.

JIC classification system is a classical method to determine the collapse risk and widely used in worldwide because it classified based on lesion extent and location of ONFH involving the lateral weightbearing surface of femoral head ${ }^{9 ; 12}$. The advantage of JIC type is its accurate prognostic value while maintaining simplicity. Several previous studies have reported that different collapse rates of ONFH using the JIC classification system, however, there are great differences with the clinical results (Table 4) 9 ; 12; ${ }^{29}$. Recently, a study with large sample data reported that five-year collapse rates of 267 necrotic femoral heads in JIC type A, B, C1, and C2 were $0 \%, 7.9 \%, 36.6 \%$, and $84.8 \%$, respectively ${ }^{12}$. Nevertheless, the present study, a total of 178 hips were followed-up for five-year and reported collapse rates of $0 \%$ in type A, $24.3 \%$ in type $B, 68.1 \%$ in type $C 1$, and $100 \%$ in type $C 2$, respectively. The reasons for this difference might resulted from the usage of combined evaluation of AP and FLL views to classify JIC types. In fact, anterior involvement of the femoral head is also an important factor of collapse ${ }^{11 ; 30}$. Previous studies in hip biomechanics have indicated that the mechanical stress is loaded on the anterolateral area of femoral head, and this area supported most of the body weight in daily activities ${ }^{31 ;} 32$. Kubo et al. demonstrated that a completed involvement of the anterior femoral head could increase the risk of collapse and necrotic femoral head with anterior area affected might collapse eventually even the necrotic lesion did not extend beyond the lateral column ${ }^{30}$. Nam et al. evaluated the fate of untreated asymptomatic ONFH with a measurement of the size of the anterolateral lesion in AP and FLL views ${ }^{33}$. Both necrotic angle and a modified kerboul method were put forward to assess collapse of the femoral head in the anterolateral weight-bearing surface of femoral head ${ }^{34 ; 35}$. Consistent with the findings of previous studies, this present study found that the collapse rate increases as the necrotic lesion on the anterolateral weight-bearing surface of femoral head becomes larger. It can provide useful clues for determining optimal treatment approaches. Type A with the lowest collapse rate is recommended for conservative treatment, and the highest collapse rate of type $\mathrm{C} 2$ is recommended to undergo jointpreserving surgery as early as possible. Although type $B$ has a certain collapse rate, conservative treatment was still recommended as most of the patient can stay painless and remain good function. The high collapse rate of type $\mathrm{C} 1$ suggests that early intervention should be determined based on whether 
necrotic lesion involved the the anterolateral weight-bearing surface of femoral head, and the precise evaluation method is yet to be further studied.

Table 4

Previous reports on the collapse rate evaluated by the Japanese Investigation Committee (JIC) classification system.

\begin{tabular}{|c|c|c|c|c|c|c|}
\hline \multirow[t]{2}{*}{ Authors (years) } & \multirow[t]{2}{*}{$\begin{array}{l}\text { Necrotic } \\
\text { hips, } \mathrm{n}\end{array}$} & \multirow[t]{2}{*}{$\begin{array}{l}\text { Mean follow-up, } \\
\text { years }\end{array}$} & \multicolumn{4}{|c|}{$\begin{array}{l}\text { Collapse rate, \% (by Kaplan-Meier surviva } \\
\text { analysis) }\end{array}$} \\
\hline & & & Type A & Type B & Type C1 & Type C2 \\
\hline $\begin{array}{l}\text { Min et al (2008) } \\
{[30]}\end{array}$ & 81 & 8.3 & 0 & 0 & 13 & 86 \\
\hline $\begin{array}{l}\text { Takashima et al } \\
\text { [9] }\end{array}$ & 86 & 10.0 & 0 & 6 & 68 & 82 \\
\hline $\begin{array}{l}\text { Kuroda et al } \\
\text { (2019) [12] }\end{array}$ & 212 & 5.0 & 0 & 8 & 37 & 85 \\
\hline $\begin{array}{l}\text { Present study } \\
\text { (2021) }\end{array}$ & 178 & 5.0 & 0 & 24 & 68 & 100 \\
\hline
\end{tabular}

In the present study, a significant difference is noted that the five year collapse rates are closely related to the existence of clinical symptoms at initial diagnosis. It is well coincided with the previous studies reporting that hip pain are strongly associated with bone marrow edema, subchondral bone fracture, and subsequent collapse in precollapse ONFH ${ }^{28 ; 36-38}$. Therefore, we suggest that hip pain may be considered as a sign for progression to advanced ONFH, even prior to collapse.

In conclusion, combined evaluation of AP and FLL views for anterolateral necrotic lesion has clinical value to predict collapse in patients with precollapse ONFH. Together by using JIC classification, it can provide an optimal choice for therapeutic strategies. Specifically, prediction of collapse and early intervention are the fundamental principles of hip preserving treatment for both systematic and asymptomatic ONFH.

\section{Declarations}

\section{Ethical Approval}

All procedures performed in studies involving human participants were in accordance with the ethical standards of the institutional and/or national research committee and with the 1964 Helsinki Declaration ant its later amendments or comparable ethical standards.

\section{Consent to Participate}

Written informed consent was obtained from individual or guardian participants. 


\section{Consent to Publish}

Written and signed consent to publish the case was obtained from the patient.

\section{Author Contributions}

Q.-S.W. carried out the experiments and data analysis and drafted and critically revised the manuscript. M.-C.H. critically revised the manuscript. T.-Y.L. revised the manuscript and provided technical assistance in preparing the manuscript. P.Y. carried out the statistical analysis. Z.-Q.C. and Q.-W.Z. supervised the project and critically revised the manuscript for important intellectual content. W.H. is the corresponding author, contributed to the study design, clinical studies, and critically revised the manuscript for important intellectual content.

\section{Funding}

This study was supported by grants from the project of the National Natural Science Foundation of China (No. 81873327, 82004392 and 81573996).

\section{Competing Interests}

The authors declare that they have no conflict of interest.

\section{Availability of data and materials}

All data or clinical images used during the study are available from the corresponding author by reasonable request.

\section{References}

1. Larson E, Jones LC, Goodman SB, et al. 2018. Early-stage osteonecrosis of the femoral head: where are we and where are we going in year 2018? International orthopaedics 42:1723-1728.

2. Zhao DW, Yu M, Hu K, et al. 2015. Prevalence of Nontraumatic Osteonecrosis of the Femoral Head and its Associated Risk Factors in the Chinese Population: Results from a Nationally Representative Survey. Chinese medical journal 128:2843-2850.

3. Boontanapibul K, Steere JT, Amanatullah DF, et al. 2020. Diagnosis of Osteonecrosis of the Femoral Head: Too Little, Too Late, and Independent of Etiology. The Journal of arthroplasty 35:2342-2349.

4. Mont MA, Salem HS, Piuzzi NS, et al. 2020. Nontraumatic Osteonecrosis of the Femoral Head: Where Do We Stand Today?: A 5-Year Update. The Journal of bone and joint surgery American volume 102:1084-1099.

5. Nishii T, Sugano N, Ohzono K, et al. 2002. Significance of lesion size and location in the prediction of collapse of osteonecrosis of the femoral head: a new three-dimensional quantification using 
magnetic resonance imaging. Journal of orthopaedic research : official publication of the Orthopaedic Research Society 20:130-136.

6. Motomura G, Yamamoto T, Yamaguchi R, et al. 2011. Morphological analysis of collapsed regions in osteonecrosis of the femoral head. The Journal of bone and joint surgery British volume 93:184-187.

7. Hu LB, Huang ZG, Wei HY, et al. 2015. Osteonecrosis of the femoral head: using CT, MRI and gross specimen to characterize the location, shape and size of the lesion. The British journal of radiology 88:20140508.

8. Wen $P$, Zhang $Y$, Hao L, et al. 2020. The effect of the necrotic area on the biomechanics of the femoral head - a finite element study. BMC musculoskeletal disorders 21:211.

9. Takashima K, Sakai T, Hamada H, et al. 2018. Which Classification System Is Most Useful for Classifying Osteonecrosis of the Femoral Head? Clinical orthopaedics and related research 476:1240-1249.

10. Cheng $W$, Xian $H$, Wang $L$, et al. 2021. Frog leg lateral view is a reliable predictor of the prognosis in osteonecrosis of the femoral head. Journal of orthopaedic research : official publication of the Orthopaedic Research Society 39:950-958.

11. Kubo Y, Motomura G, Ikemura S, et al. 2018. The effect of the anterior boundary of necrotic lesion on the occurrence of collapse in osteonecrosis of the femoral head. International orthopaedics 42:14491455.

12. Kuroda Y, Tanaka T, Miyagawa T, et al. 2019. Classification of osteonecrosis of the femoral head: Who should have surgery? Bone \& joint research 8:451-458.

13. Daniel M, Herman S, Dolinar D, et al. 2006. Contact stress in hips with osteonecrosis of the femoral head. Clinical orthopaedics and related research 447:92-99.

14. Gao F, Han J, He Z, et al. 2018. Radiological analysis of cystic lesion in osteonecrosis of the femoral head. International orthopaedics 42:1615-1621.

15. Liu GB, Li R, Lu Q, et al. 2020. Three-dimensional distribution of cystic lesions in osteonecrosis of the femoral head. Journal of orthopaedic translation 22:109-115.

16. Shi S, Luo P, Sun L, et al. 2021. Prediction of the progression of femoral head collapse in ARCO stage 2-3A osteonecrosis based on the initial bone resorption lesion. The British journal of radiology 94:20200981.

17. Sun W, Li ZR, Wang BL, et al. 2014. Relationship between preservation of the lateral pillar and collapse of the femoral head in patients with osteonecrosis. Orthopedics 37:e24-28.

18. Nagoya S, Nagao M, Takada J, et al. 2004. Predictive factors for vascularized iliac bone graft for nontraumatic osteonecrosis of the femoral head. Journal of orthopaedic science : official journal of the Japanese Orthopaedic Association 9:566-570.

19. Wei QS, Zhang QW, He W, et al. 2018. Clinical significance for new type of necrotic femoral head based on X- ray. Chinese Journal of Joint Surgery 12:8. 
20. Wei QS, Fang B, Chen ZQ, et al. 2019. Role of bone status in anterolateral portion of femoral head in the progression of osteonecrosis of the femoral head. Zhongguo Zuzhi Gongcheng Yanjiu 23:7.

21. Yoon BH, Mont MA, Koo KH, et al. 2020. The 2019 Revised Version of Association Research Circulation Osseous Staging System of Osteonecrosis of the Femoral Head. The Journal of arthroplasty 35:933-940.

22. Konan S, Rayan F, Haddad FS. 2010. Is the frog lateral plain radiograph a reliable predictor of the alpha angle in femoroacetabular impingement? The Journal of bone and joint surgery British volume 92:47-50.

23. Hungerford DS, Jones LC. 2004. Asymptomatic osteonecrosis: should it be treated? Clinical orthopaedics and related research:124-130.

24. Mont MA, Zywiel MG, Marker DR, et al. 2010. The natural history of untreated asymptomatic osteonecrosis of the femoral head: a systematic literature review. The Journal of bone and joint surgery American volume 92:2165-2170.

25. Hernigou P, Habibi A, Bachir D, et al. 2006. The natural history of asymptomatic osteonecrosis of the femoral head in adults with sickle cell disease. The Journal of bone and joint surgery American volume 88:2565-2572.

26. Zhu L, Han J, Guo R, et al. 2020. An Automatic Classification of the Early Osteonecrosis of Femoral Head with Deep Learning. Current medical imaging 16:1323-1331.

27. Wu W, He W, Wei QS, et al. 2018. Prognostic analysis of different morphology of the necrotic-viable interface in osteonecrosis of the femoral head. International orthopaedics 42:133-139.

28. Hatanaka H, Motomura G, Ikemura S, et al. 2019. Differences in magnetic resonance findings between symptomatic and asymptomatic pre-collapse osteonecrosis of the femoral head. European journal of radiology 112:1-6.

29. Min BW, Song KS, Cho CH, et al. 2008. Untreated asymptomatic hips in patients with osteonecrosis of the femoral head. Clinical orthopaedics and related research 466:1087-1092.

30. Kubo Y, Motomura G, Ikemura S, et al. 2020. Effects of anterior boundary of the necrotic lesion on the progressive collapse after varus osteotomy for osteonecrosis of the femoral head. Journal of orthopaedic science : official journal of the Japanese Orthopaedic Association 25:145-151.

31. Yoshida H, Faust A, Wilckens J, et al. 2006. Three-dimensional dynamic hip contact area and pressure distribution during activities of daily living. Journal of biomechanics 39:1996-2004.

32. Genda E, Iwasaki N, Li G, et al. 2001. Normal hip joint contact pressure distribution in single-leg standing-effect of gender and anatomic parameters. Journal of biomechanics 34:895-905.

33. Nam KW, Kim YL, Yoo JJ, et al. 2008. Fate of untreated asymptomatic osteonecrosis of the femoral head. The Journal of bone and joint surgery American volume 90:477-484.

34. Kerboul M, Thomine J, Postel M, et al. 1974. The conservative surgical treatment of idiopathic aseptic necrosis of the femoral head. The Journal of bone and joint surgery British volume 56:291296. 
35. Ha YC, Jung WH, Kim JR, et al. 2006. Prediction of collapse in femoral head osteonecrosis: a modified Kerboul method with use of magnetic resonance images. The Journal of bone and joint surgery American volume 88 Suppl 3:35-40.

36. Meier R, Kraus TM, Schaeffeler C, et al. 2014. Bone marrow oedema on MR imaging indicates ARCO stage 3 disease in patients with AVN of the femoral head. Eur Radiol 24:2271-2278.

37. Koo KH, Ahn IO, Kim R, et al. 1999. Bone marrow edema and associated pain in early stage osteonecrosis of the femoral head: prospective study with serial MR images. Radiology 213:715-722.

38. Ito $\mathrm{H}$, Matsuno T, Minami A. 2006. Relationship between bone marrow edema and development of symptoms in patients with osteonecrosis of the femoral head. AJR American journal of roentgenology 186:1761-1770.

\section{Figures}

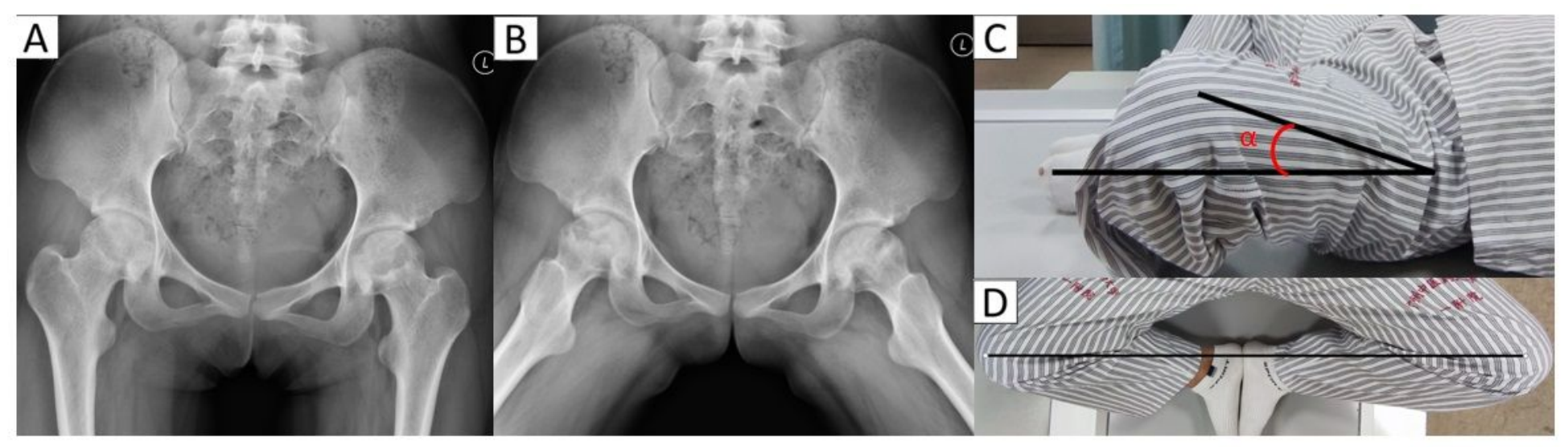

Figure 1

(A, B) A typical AP and FLL view of bilateral hip. (C, D) A standard filming feature of the FLL view: patients were obtained in a supine position with the bilateral hips and knees flexed and the feet contacted close to each other; the thigh was abducted and externally rotated at a degree of $30^{\circ}$ while ensuring that the plane of the pelvis was parallel to the plane of the $x$-ray table. $a=30^{\circ}$. 

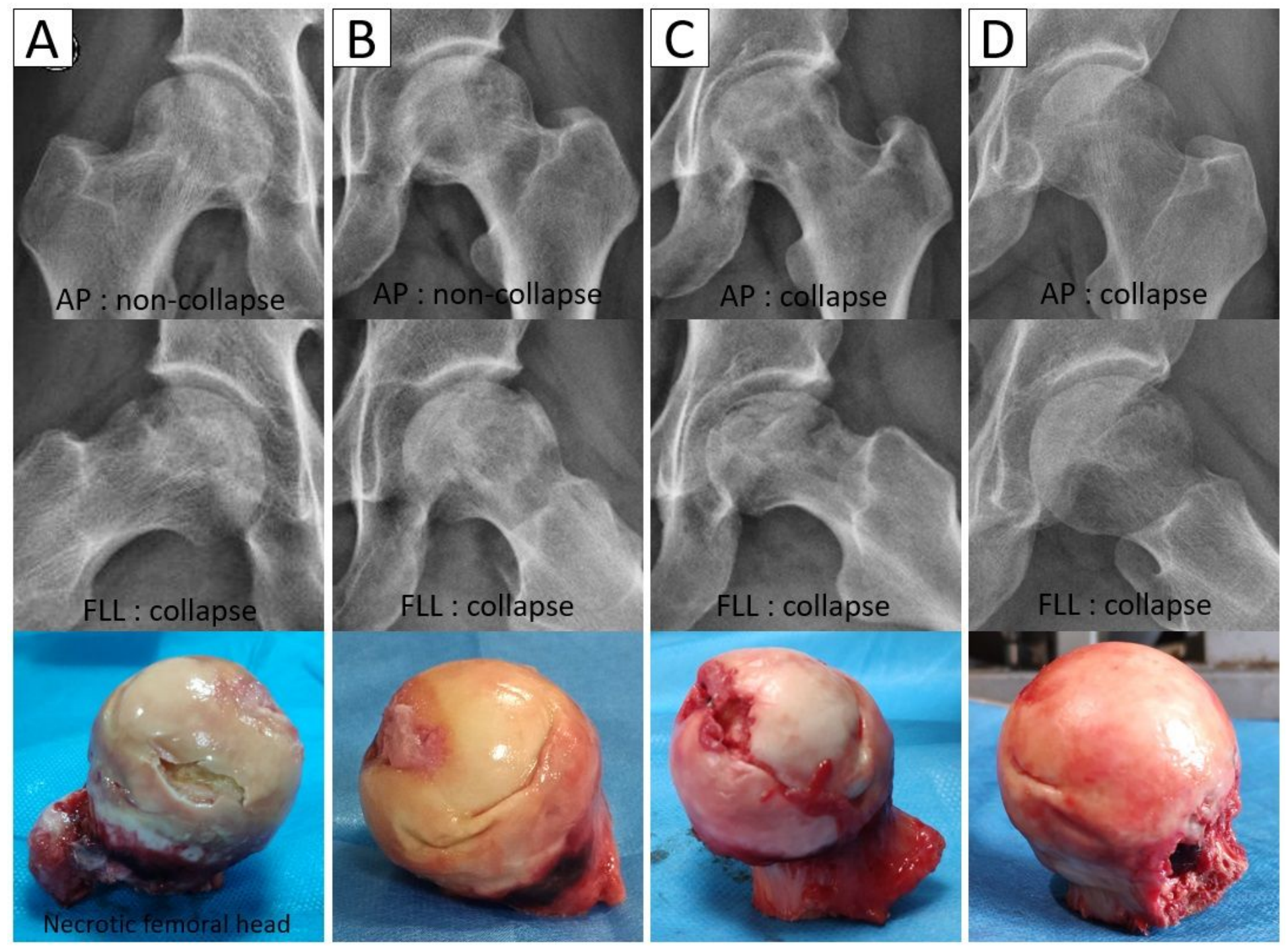

\section{Figure 2}

Five necrotic femoral heads shown on AP and FLL views. (A) a 63-year-old man with alcohol induced$\mathrm{ONFH}$, and (B) a 40-year-old man with idiopathic ONFH, both showed non-collapse of the femoral head on AP view and collapse on FLL view. Corresponding the gross specimen photograph showed cartilage defect or folds at the anterior portion of the femoral head. (C) a 32-year-old woman with steroid inducedONFH, and (D) a 49-year-old man with alcohol induced-ONFH, both showed collapse of the femoral head on AP and FLL views, and corresponding the gross specimen photograph showed cartilage folds at the anterolateral portion of the femoral head. ONFH: osteonecrosis of femoral head; AP: anteroposterior view; FLL『frog-leg lateral view. 

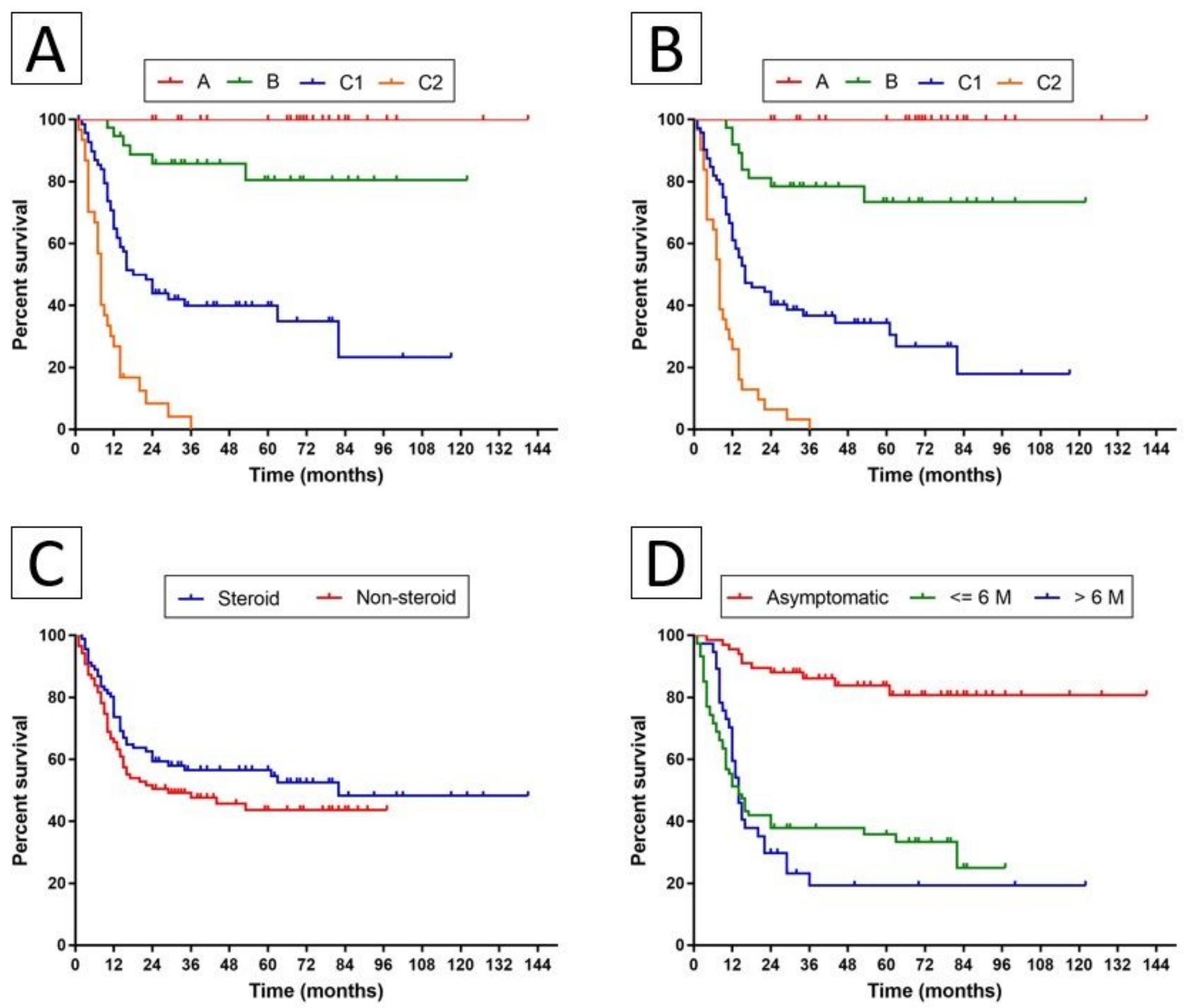

Figure 3

Kaplan-Meier survival curves of patients with precollapse ONFH. (A) the cumulative five-year collapse rates were indicated as follows: using the JIC type on AP view alone, type $A, 0 \%$; type $B, 16.2 \%$; type $C 1$, $58.3 \%$; and type $C 2,100 \%$ ( $p<0.001$, log-rank test). (B) collapse rates were evaluated on AP and FLL views as follows: type $A, 0 \%$; type $B, 24.3 \%$; type $C 1,68.1 \%$; and type $C 2,100 \%$ ( $<<0.001$, log-rank test). (C) collapse rate according to steroid use; there was no difference in terms of time to collapse $(p=0.186, \log$ rank test). (D) collapse rate according to symptomatic or asymptomatic; there was a significant difference in terms of time to collapse ( $<<0.001$, log-rank test). ONFH: osteonecrosis of femoral head; AP: anteroposterior view; FLL冈frog-leg lateral view. 


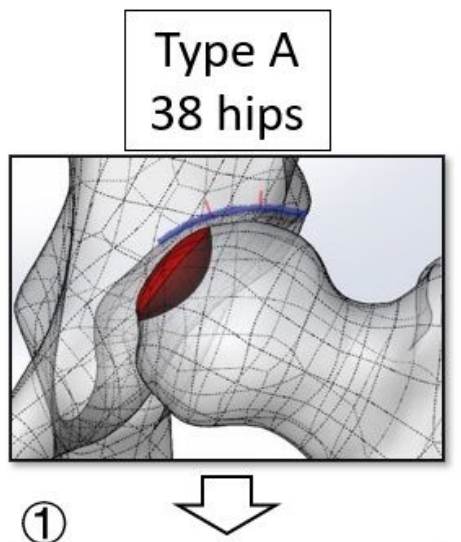

1-yr collapse rate $0 \%$ 2-yr collapse rate $0 \%$ 5 -yr collapse rate $0 \%$

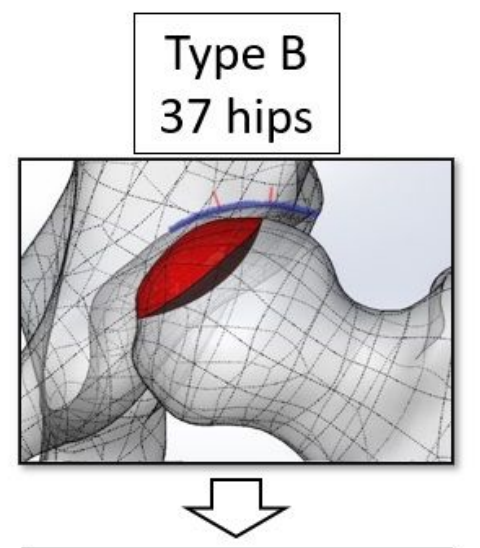

1-yr collapse rate $5.4 \%$ 2 -yr collapse rate $13.5 \%$ 5 -yr collapse rate $16.2 \%$

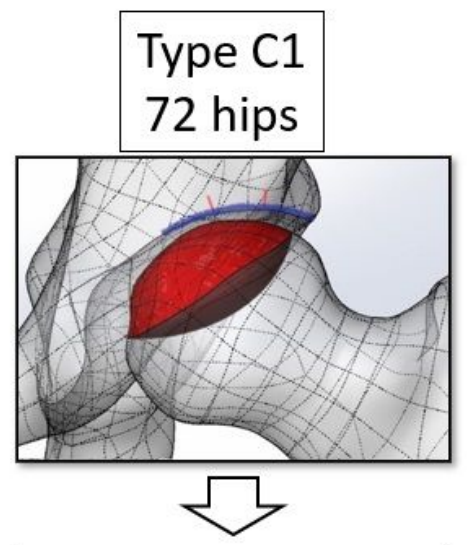

1-yr collapse rate $33.3 \%$ 2-yr collapse rate $52.8 \%$ 5 -yr collapse rate $58.3 \%$

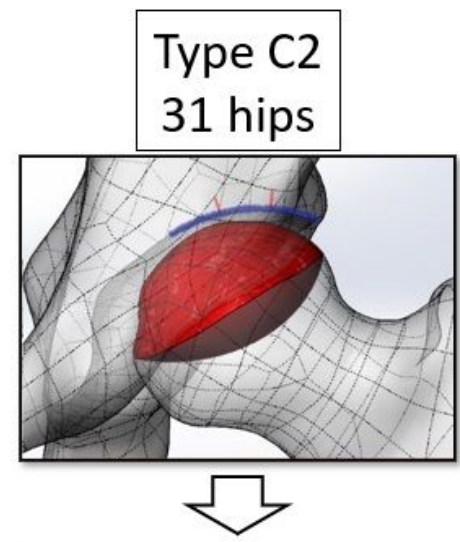

1-yr collapse rate $71.0 \%$ 2-yr collapse rate $87.1 \%$ 5 -yr collapse rate $100 \%$
(2)

1-yr collapse rate $0 \%$ 2-yr collapse rate $0 \%$

5 -yr collapse rate $0 \%$ 1-yr collapse rate $8.1 \%$ 2-yr collapse rate $21.6 \%$ 5 -yr collapse rate $24.3 \%$

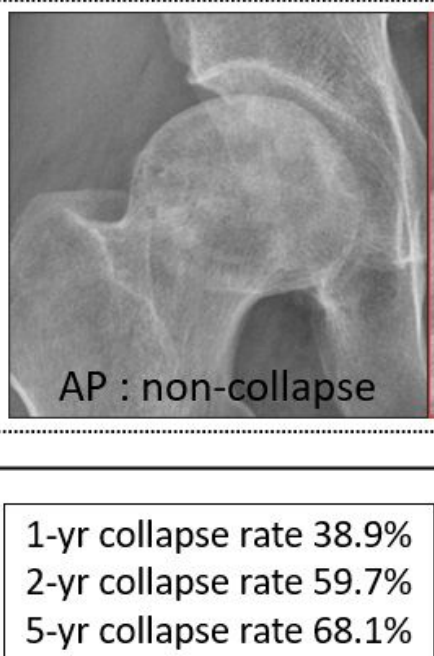

1-yr collapse rate $74.2 \%$ 2-yr collapse rate $93.5 \%$

5 -yr collapse rate $100 \%$

\section{Figure 4}

Five-year collapse rates of each JIC type as evaluated by the cox proportional hazards regression model. JIC types on AP view identify that the greater the lateral involvement of the necrotic lesion, the higher the risk of collapse. A higher collapse rate can be seen on AP and FLL views, which simultaneously identified the collapse of anterior and lateral location of femoral head. yr: year; AP: anteroposterior view; FLL囚frogleg lateral view. 